\title{
APLICACIÓN DEL RIEGO DEFICITARIO CONTROLADO EN DURAZNERO (PRUNUS PÉRSICA BATSCH) BAJO EL SISTEMA DE RIEGO POR GOTEO
}

\author{
APPLICATION OF CONTROLLED DEFICIT IRRIGATION IN PEACH (PRUNUS PERSICA \\ BATSCH) UNDER THE DRIP IRRIGATION SYSTEM
}

\author{
${ }^{1}$ Celia Vásquez Samaniego, ${ }^{2}$ Miguel Angel Sánchez Delgado y ${ }^{3}$ Alejandro Pacheco Avalos
}

\begin{abstract}
Resumen
El presente trabajo se realizó en la Universidad Nacional Agraria La Molina con la finalidad de evaluar el efecto del riego deficitario controlado sobre la producción del cultivo de durazno. Se aplicaron dos tratamientos de riego, un tratamiento control (C) equivalente al 100\% de la ETc y un tratamiento de riego deficitario controlado (RDC) en el que se utilizó el 75\% de la ETc en la primera fase de fructificación, 50\% de la ETc en la fase de endurecimiento del carozo y $100 \%$ de la ETc en la tercera fase de fructificación. Los resultados indican que no se detectaron diferencias significativas entre el tratamiento control y el RDC en el crecimiento vegetativo de las plantas (diámetro de ramas, altura de planta y diámetro de copa) ni en el crecimiento reproductivo
\end{abstract}

Palabras clave: Riego deficitario controlado, riego por goteo, durazno.

\begin{abstract}
This work was performed at National Agrarian University of La Molina, Lima. The objective of this study was to investigate the effect of regulated deficit irrigation with drip irrigation techniques for peach trees (Prunus persica). The evaluated treatments were: Control (C): control with daily irrigation equivalent to $100 \%$ of the crop evapotranspiration (ETc); RDI (regulated deficit irrigation), which is irrigated with $75 \%$ of the ETC in the first period of fructification; $50 \%$ of ETc in the hardening phase of bone and $100 \%$ ETc in the third period of fructification. Results showed no significant differences between control treatment and RDI in both the vegetative growth of the plants (diameter branches, plant height and crown diameter) and reproductive growth.
\end{abstract}

Keys words: Regulated deficit irrigation, drip irrigation, peach trees

\section{Introducción}

La racionalización del uso del agua se está convirtiendo en un objetivo importante para muchos sectores de nuestra sociedad (industria, servicios, urbano, administración, agrícola) y en fuente de debate en diferentes esferas del ámbito social, político y económico.

En los últimos años se ha asistido a un importante incremento de trabajos publicados que abarcan la temática del riego deficitario y su efecto sobre la producción en árboles frutales; no obstante, algunas de estas técnicas o estrategias aplicadas a otros medios no obtienen los resultados esperados debido a las condiciones diversas en las que se aplica. Es por ello que el estudio del comportamiento de resultados es muy útil para la agricultura de cada zona.
El Riego Deficitario Controlado (RDC) es una alternativa en situaciones de limitada disponibilidad de agua para riego. Martínez (2007) define al RDC como "la práctica de regular y restringir el agua de riego empleada en un cultivo, tratando de aplicarla en los momentos en los que su eficacia sea máxima, de acuerdo con los objetivos del cultivo". Por otro lado Sánchez y Torrecillas (1995) indican que el RDC se basa en reducir los aportes hídricos en aquellos periodos fenológicos considerados no críticos para la producción y calidad de la cosecha y cubrir plenamente la demanda de la planta durante el resto del ciclo del cultivo.

Los trabajos de RDC en melocotonero se han hecho mayoritariamente en variedades tempranas y medias y los resultados en general muestran efectos beneficiosos en el control del crecimiento vegetativo. En árboles jóvenes de melocotonero temprano, tras dos años de experimento,

\footnotetext{
${ }^{1}$ Ingeniero Agrícola

${ }^{2}$ Dpto. de Recursos Hídricos. Facultad Ingeniería Agrícola - UNALM E-mail: msanchez@lamolina.edu.pe

${ }^{3}$ Dpto. de Horticultura. Facultad Agronomía - UNALM E-mail: apachec@lamolina.edu.pe
} 
Alarcón et al., (2006) concluyeron que los diferentes tratamientos deficitarios no tuvieron efectos significativos sobre la producción total obtenida, asimismo todos los tratamientos deficitarios supusieron una mejora en la eficiencia en el uso de agua, siendo el riego deficitario controlado el que ofreció unos valores de eficiencia más elevados, permitiendo ahorros de agua cercanos al $60 \%$ sin reducciones significativas de la producción.

Es importante resaltar que el Riego Deficitario Controlado (RDC), aplicados en distintas zonas del mundo, produce un ahorro de agua de riego, un aumento de la eficiencia en el uso del agua y una reducción del vigor vegetativo de los árboles frutales de forma que los rendimientos se mantienen o incluso aumentan.

El presente trabajo tiene como objetivo general comprobar mediante la aplicación del Riego Deficitario Controlado (RDC), el efecto en la producción de duraznero 'Canario' y 'Florida 39'. Se pretende además evaluar el efecto de restricción hídrica sobre el crecimiento vegetativo (altura, diámetro de copa y sección transversal de ramas) y crecimiento reproductivo (crecimiento y desarrollo del fruto).

\section{Materiales y métodos}

La investigación se realizó en la parcela experimental de duraznero de la Facultad de Ingeniería Agrícola (FIA), perteneciente a la Universidad Nacional Agraria La Molina. La parcela experimental está dividida en dos sectores, en el primer sector se encuentra instalada la variedad Florida 39 y en el segundo, la variedad Canario, ambas variedades de comportamiento temprano (cosecha a principios de diciembre). Encontrándose reinjertados sobre pies francos de duraznero Okinawa. La edad del reinjerto es de 2 años, se emplearon un total de 26 árboles, seis pares para el primer sector y siete pares para el segundo.

El desarrollo del proyecto se realizó bajo la modalidad del tipo experimental de campo, a través de la aplicación de dos tratamientos de riego:

- C, riego controlado, reponiendo el $100 \%$ de la evapotranspiración de cultivo.

- $\mathrm{RDC}$, riego deficitario controlado en el que se riega aplicando diferentes porcentajes de ETc: inicio de la campaña, floración y fase I de fructificación con el $75 \%$ de la ETc; fase de endurecimiento de hueso que se regó con el 50\% de la ETc y la fase III de fructificación (rápido crecimiento) regado con el 100\% de la ETc.

Se utilizó un diseño experimental completamente randomizado con 7 repeticiones para la variedad "Florida 39 " y 6 repeticiones para la variedad "Canario" con la finalidad de indicar dentro de un rango de probabilidad el efecto de los diferentes riegos en el rendimiento del cultivo. Cada unidad experimental fue definida por dos plantas.
El método de riego utilizado fue por goteo. En el mes de febrero del 2010 se sustituyeron los goteros existentes por goteros autocompensados Irritec de 4 1/s dado la antigüedad de los primeros y la desuniformidad de caudal de emisión de los mismos.

Antes de comenzar los riegos en la estación de crecimiento se evaluó el coeficiente de variación de fabricación $(\mathrm{CVF})$, Se puede señalar que el gotero IRRITEC presentó un valor igual a $7.83 \%$ de CVF, por lo tanto con este emisor no se alcanzó altos niveles de uniformidad en instalaciones de riego localizado, este emisor es catalogado como B por la norma internacional ISO 9260. Para la norma ASAE, que también posee parámetros para clasificar a emisores de riego según su coeficiente de variación de fabricación, considera al emisor IRRITEC en la categoría "MARGINALES". Se determinó la curva caudal - presión, se tomaron muestras representativas de los emisores Irritec, los cuales fueron sometidos a diferentes presiones, a fin de obtener los caudales correspondientes a cada presión. En base a la presión mínima y máxima se definió el número de presiones de prueba obteniendo como mínimo $10 \mathrm{cb}$ y como máximo de $20 \mathrm{cb}$ de presión. Es decir, dentro de ese rango de presión, los goteros emitirán un caudal con mayor posibilidad de lograr la uniformidad adecuada en el riego; asimismo, se evaluó la uniformidad de riego del sistema siguiendo la metodología propuesta por Merriam y Keller (1978). El caudal medio erogado por los goteros fue de $4.87 \mathrm{~L} \mathrm{~h}^{-1}$, con un coeficiente de uniformidad de 82.67 , encontrándose dentro de los límites recomendados para las características del sistema.

Para el manejo del riego en los tratamientos de riego deficitario y control se instaló en los anillos (manguera de polietileno de $16 \mathrm{~mm}$ ) una válvula ramal de $16 \mathrm{~mm}$, que permitió interrumpir el riego sin interferir con la operación normal de la plantación.

La duración de la etapa fenológica abarcó desde la floración hasta la cosecha (150 a 180 días). Las principales labores del manejo realizadas fueron: poda, para eliminar ramas verticales, aplicación de fertilizantes al suelo y por vía foliar (micronutrientes quelatados), y la eliminación de maleza. Los árboles fueron sometidos a un aclareo manual de frutos durante la segunda semana de octubre del 2010, que como se comprobó más tarde no fue uniforme entre tratamientos.

La fertilización para la variedad "Florida 39" se realizó en los meses de octubre, noviembre, diciembre y enero con una frecuencia de quince días. Para la variedad "Canario", la fertilización se realizó los meses de octubre, noviembre y diciembre cada quince días. La dosis de fertilización aplicada fue de 110-40-120 (N, P, K) $10 \mathrm{Ca}-40 \mathrm{Mg}$, las cuales se fraccionaron en ocho y seis aplicaciones para las variedades Canario y Florida 39 respectivamente que se inyectaron al sistema riego por ferti-irrigación. 
El control fitosanitario fue cultural y químico, con aplicaciones de productos químicos para el control de plagas y enfermedades y las malezas se controlaron mediante métodos físicos: arranque manual, escarda con azada, corte con machete u otra herramienta y labores de cultivo. Las condiciones del manejo de la parcela pueden ser consideradas casi óptimas y no fueron un factor de variación en el experimento.

Durante la estación de crecimiento 2010-2011 se realizó la caracterización fenológica del duraznero, en cada tratamiento se registró fechas por estación de crecimiento: inicio, plenitud y fin de floración, inicio de fructificación y maduración de frutos, con la definición de los estados fenológicos y reproductivos.

El crecimiento vegetativo se controló con la medida del crecimiento de: perímetro de ramas (longitud), copa (anchura) y mediciones de la altura de las plantas para los dos tratamientos.

La producción se determinó pesando el total de frutos en cada recolección en seis y siete árboles por tratamiento para las variedades "Canario" y "Florida 39 " respectivamente. Se hicieron 2 determinaciones del perímetro de la rama (marzo 2010 y marzo 2011) para evaluar el efecto que el aporte hídrico diferencial de los tratamientos pudiera tener sobre el crecimiento del árbol y se calculó la sección transversal de la rama (STR).

Luego del período de floración, transcurrida la polinización de flores y luego de la primera caída natural de flores, se contó el número de frutos.

La curva característica del crecimiento del fruto se determinó a través del diámetro ecuatorial marcándose cinco frutos por árbol elegidos al azar. Para la variedad "Florida 39", el diámetro ecuatorial de cada fruto ( $\mathrm{mm})$ fue medido con un vernier convencional, cada uno con una frecuencia de nueve a once días a partir del 27 de octubre hasta el 12 de diciembre de 2010. Para la variedad "Canario" se comenzó a medir a partir del 28 de setiembre de 2010 hasta el 02 de enero de 2011. El diámetro ecuatorial es el diámetro mayor y es el que se considera para la clasificación comercial de frutos según su calibre.

La cosecha se realizó los días 19, 22, 26 de diciembre de 2010 y 02 enero de 2011 para los frutos de la variedad "Florida 39"; y para la variedad "Canario" los días 15, 18, 20,22 y 25 de enero del 2011. En cada una de las cosechas se calculó el rendimiento promedio por planta ( $\mathrm{kg} / \mathrm{planta})$ y número de frutos por árbol. Tomando en consideración el diámetro ecuatorial de los frutos, los cuales fueron contados y pesados. También se clasificó la producción según su calibre en cinco categorías: Categoría 1, frutos mayores de $5.0 \mathrm{~cm}$ de diámetro; categoría 2, frutos de 4.40 a $5 \mathrm{~cm}$ de diámetro; categoría 3 , frutos de diámetro de 3.8 a $4.3 \mathrm{~cm}$; categoría 4 , frutos con diámetros de 2.5 a $3.7 \mathrm{~cm}$; y la categoría 5 que incluye a toda fruta con daño físico o biológico.

\section{Resultados y discusión \\ Volumen de agua utilizado}

La Fig. 1 muestra los valores diarios de ETo, ETc y agua aportada por riego para los diferentes cultivares respectivamente. Los valores de ETo corresponden a la media histórica de la zona. La diferencia entre la curva de la ETo, tomada de los datos de la estación meteorológica y la ETc fue debido al valor de coeficiente de cultivo (Kc) considerado. A partir de mediados de octubre, la ETc aumentó en coincidencia con el crecimiento de brotes, y las lluvias fueron casi ausentes, la máxima ETc se registró en el mes de diciembre y fue de 2,64 mm/día, el promedio de evapotranspiración diaria para ese mes fue de $3,37 \mathrm{~mm}$ diarios.
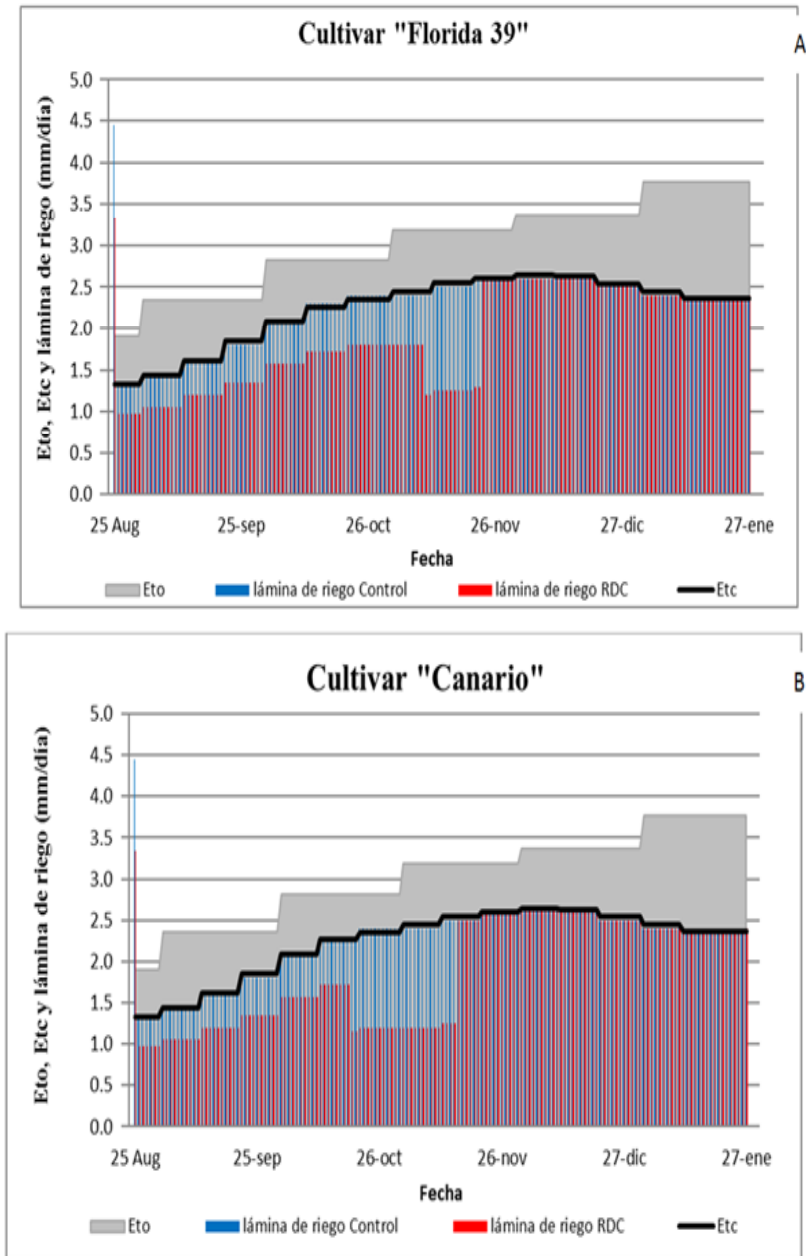

Figura 1. Evapotranspiración semanal de referencia (ETo) del cultivo (ETc) y lámina de riego aplicada por día en el tratamiento Control y RDC para las variedades "Florida 39" (A) y "Canario" (B). 
Se puede observar en la figura que el día 25 de agosto se regó con una lámina de riego mucho mayor puesto que el riego anterior (riego pesado) se llevó a cabo el 17 de agosto, es decir una semana anterior. El 25 de agosto del año 2010 se dio inicio a la aplicación del RDC al 75\% de la ETc durante el periodo de brotación, floración y primera etapa de fructificación o crecimiento moderado para la variedad "Canario" como para "Florida 39" (Tabla 1).

Tabla 1. Consumo de agua de riego en función de las fases de crecimiento del fruto.

\begin{tabular}{lcccccc}
\hline Tratamientos & \multicolumn{3}{c}{$\begin{array}{c}\text { Fases de crecimiento del } \\
\text { fruto } \\
\text { días del año }\end{array}$} & & \multicolumn{2}{c}{$\begin{array}{c}\text { Agua aplicada en } \\
\text { riego }\end{array}$} \\
\hline Canario & I & II & III & PC & $\begin{array}{c}\text { Total } \\
(\mathrm{mm})\end{array}$ & $\%$ Control \\
CC - Control & & & & & 369.111 & $100 \%$ \\
CD - RDC & $22-55$ & $55-82$ & $82-143$ & ---- & 316.667 & $86 \%$ \\
Florida 39 & & & & & & \\
FC - Control & & & & & & \\
FD - RDC & 46-76 & $76-90$ & $90-117$ & $131-156$ & 369.111 & $100 \%$ \\
\end{tabular}

Durante todo el proceso de crecimiento moderado de la variedad "Canario", los árboles bajo ensayo recibieron un total de $71.167 \mathrm{~mm}$ de riego concluyendo la aplicación el 18 de octubre del mismo año. En el caso de la variedad "Florida 39", la Fase I finalizó el 08 de noviembre del 2010 recibiendo un total de $120.05 \mathrm{~mm}$ (Tabla 2).

La Fase II para la variedad Florida 39 inició el 19 de octubre del 2010. Aquí se aplicó el RDC al 50\% de la ETc; durante el proceso de crecimiento lento que duró veintisiete días, la planta recibió un total de $42.389 \mathrm{~mm}$ de riego. Para la variedad Canario la Fase II inició el 09 de noviembre del 2010, al igual que en la variedad Florida 39, se aplicó el RDC al 50\% de la ETc durante todo el proceso de crecimiento lento; no obstante éste duró catorce días, recibiendo un total de $12.278 \mathrm{~mm}$ de riego.

Las cantidades totales de agua de riego aplicados al tratamiento Control para ambas variedades (CC y FC) fue de $369.111 \mathrm{~mm}$, mientras que para el tratamiento deficitarios se aplicó $303.0 \mathrm{~mm}$ en la variedad "Florida 39 " (82\% del tratamiento control) y $316.67 \mathrm{~mm}$ en la variedad "Canario" (86\% del tratamiento control) (Tabla 2).

Tabla 2. Volumen de agua aplicado (m3 ha-1 año-1) en cada tratamiento.

\begin{tabular}{|c|c|c|c|c|c|c|c|c|}
\hline \multirow{2}{*}{ Tratamiento } & \multirow{2}{*}{$\mathrm{T}-75 \%$} & \multicolumn{3}{|c|}{ Fase de crecimiento del fruto } & \multirow{2}{*}{ Cosecha } & \multirow{2}{*}{ Poscosecha } & \multirow{2}{*}{$\begin{array}{c}\text { Total } \mathrm{m}^{3} / \\
\text { ha }\end{array}$} & \multirow{2}{*}{$\begin{array}{c}\% \text { de ahorro de } \\
\text { agua }\end{array}$} \\
\hline & & $\mathrm{I}$ & II & III & & & & \\
\hline \multicolumn{9}{|l|}{ Control } \\
\hline Canario & 255.56 & 693.3 & 847.8 & 1627.8 & 266.67 & & 3691.1 & 0 \\
\hline Florida & 781.11 & 825.6 & 245.6 & 808.9 & 224.4 & 805.6 & 3691.1 & 0 \\
\hline \multicolumn{9}{|l|}{$\mathrm{RDC}$} \\
\hline Canario & 191.67 & 520 & 423.9 & 1627.8 & 266.67 & & 3166.7 & 14 \\
\hline Florida & 585.83 & 619.2 & 122.8 & 808.9 & 224.4 & 805.6 & 3030 & 18 \\
\hline
\end{tabular}

\section{Registros fenológicos}

Durante el año de seguimientos fenológico de las dos variedades de durazno (Canario y Florida 39), el ritmo de transición y desarrollo de las etapas fenológicas muestra un adelantamiento significativo en la variedad Canario (Tabla 3).

Tabla 3. Comportamiento fenológico de las variedades de durazno (Prunus pérsica)- Canario y Florida 39.

\begin{tabular}{lccccccc}
\hline \multirow{2}{*}{ Variedad } & A. & $\begin{array}{c}\text { B. } \\
\text { Yema }\end{array}$ & $\begin{array}{c}\text { C. } \\
\text { Botón } \\
\text { rosado }\end{array}$ & $\begin{array}{c}\text { D. } \\
\text { Inicio de } \\
\text { floración }\end{array}$ & $\begin{array}{c}\text { E. } \\
\text { Plena } \\
\text { floración }\end{array}$ & $\begin{array}{c}\text { F. } \\
\text { Caída de restos } \\
\text { florales }\end{array}$ & $\begin{array}{c}\text { G. } \\
\text { Pruto } \\
\text { Pequeño }\end{array}$ \\
\hline Canario & $25 / 07 / 2010$ & $08 / 08 / 2010$ & $19 / 08 / 2010$ & $27 / 08 / 2010$ & $05 / 09 / 2010$ & $10 / 09 / 2010$ & $15 / 09 / 2010$ \\
Florida 39 & $10 / 08 / 2010$ & $25 / 08 / 2010$ & $02 / 09 / 2010$ & $09 / 09 / 2010$ & $22 / 09 / 2010$ & $01 / 10 / 2010$ & $09 / 10 / 2010$ \\
\hline
\end{tabular}

El posterior desarrollo y evolución de los estados fenológicos, mostró un adelantamiento en la variedad Canario. Esta diferencia se visualizó en la transición del estado de "Caída de restos florales" al estado de "Fruto pequeño". A esta fecha, la variedad antes mencionada presentó un adelantamiento alrededor de 30 días con respecto a la variedad Florida 39.
En relación con el periodo de cosecha, se produjo un cambio rotundo con respecto a la tendencia de crecimiento indicado anteriormente, en donde se puede observar que la cosecha de la variedad Florida 39 se realizó la segunda quincena de diciembre, mientras que la variedad Canario, se realizó entre el 15 y 25 del mes de enero (Tabla 4). 
Tabla 4. Datos de quienes establecimiento y producción de las variedades de durazno (Prunus pérsica)- Canario y Florida 39.

\begin{tabular}{lcccc}
\hline Variedad & Año de establecimiento & $\mathrm{N}^{\circ}$ Plantas & Fecha de cosecha & Producción (Kg/planta) \\
\hline Canario & 2009 & 28 & $15 / 01 / 11$ a 25/01/11 & 26.9 \\
Florida 39 & 2009 & 47 & $19 / 12 / 10$ a 02/01/11 & 8.3 \\
\hline
\end{tabular}

\section{CRECIMIENTO VEGETATIVO}

\section{Sección Transversal de las Ramas principales (STR)}

El crecimiento vegetativo del árbol fue controlado a través de la sección transversal de las ramas, medido luego de los tratamientos de déficit a $5 \mathrm{~cm}$ arriba del injerto. Aunque en las tres ocasiones en que se determinó los valores de este parámetro, no se encontraron diferencias significativas entre tratamientos de riego dentro de una misma variedad. Sin embargo, se detectaron diferencias numéricas muy pequeñas tanto para la variedad Florida
39 y Canario en los tratamientos DCR en comparación con los controlados. Por otro lado en el Tabla 5 se presenta el porcentaje de sección transversal para los dos tratamientos en cada variedad. Donde se puede observar que la variedad Canario presenta un $97.02 \%$ respecto al testigo de crecimiento radial luego de la temporada de restricción hídrica. En duraznero de maduración temprana con RDC poscosecha Larson et al., (1988) determinaron que el crecimiento radial luego de una temporada de déficit fue del $71 \%$ respecto al testigo.

Tabla 5. Sección transversal de ramas (STR) de durazno "Canario" y "Florida 39" producido durante el ciclo de cultivo en el que se realizó el ensayo.

\begin{tabular}{|c|c|c|c|c|}
\hline & & PR 02/10 AV & PR 03/11 AV & $\%$ Con respecto al testigo \\
\hline \multirow{2}{*}{ Florida 39} & FD - RDC & $8.77 \mathrm{a}^{\mathrm{z}}$ & $34.82 \mathrm{a}^{\mathrm{z}}$ & 94.21 \\
\hline & FC - Control & $9.78 \mathrm{a}$ & $36.96 \mathrm{a}$ & 100 \\
\hline CV \% & & 11.42 & 5.41 & \\
\hline \multirow{2}{*}{ Canario } & $\mathrm{CD}-\mathrm{RDC}$ & $11.78 \mathrm{a}^{\mathrm{z}}$ & $34.32 \mathrm{a}^{\mathrm{z}}$ & 97.02 \\
\hline & CC - Control & $13.28 \mathrm{a}$ & $35.37 \mathrm{a}$ & 100 \\
\hline CV \% & & 5.85 & 5.98 & \\
\hline
\end{tabular}

Cada valor representa la media de 6 repeticiones, en las que se midieron los perímetros de rama de 8 árboles.

PR 02/10 AV ...... Sección transversal de rama $\left(\mathrm{dm}^{2}\right)$ medido en febrero de 2010.

PR 03/11 ${ }^{\mathrm{AV}}$...... Sección transversal de rama $\left(\mathrm{dm}^{2}\right)$ medido en marzo de 2011.

$\mathrm{CV}$ : coeficiente de variación

${ }^{\mathrm{z}}$ Valores con diferentes letras dentro de la fila indica diferencias significativas para $\alpha=0.05$.

AV Análisis según Proc ANOVA.

\section{Altura y ancho de las plantas}

Al analizar estadísticamente la altura y el ancho de copa de las plantas, resultó que para las variedades "Florida 39" y "Canario" el tratamiento control no se diferenció significativamente del RDC (Tabla 6). Dicho resultado se debió principalmente a que, en la etapa de mayor desarrollo vegetativo, la dosis de riego solo se diferenció en $14 \%$ y $18 \%$ para las variedades Canario y Florida 39 respectivamente, lo que no implicó alteración en el crecimiento de las plantas.

Tabla 6. Altura máxima (m) y ancho de copa (m) para las variedades Canario y Florida 39.

\begin{tabular}{lccc}
\hline & Tratamientos & $\begin{array}{c}\text { Altura } \\
\text { MÁX }\end{array}$ & $\begin{array}{c}\text { Ancho de } \\
\text { Copa }\end{array}$ \\
\hline Florida 39 & FD - RDC & $2.43 \mathrm{a}^{\mathrm{z}}$ & $3.18 \mathrm{a}^{\mathrm{z}}$ \\
& FC - Control & $2.65 \mathrm{a}$ & $33.36 \mathrm{a}$ \\
$\mathrm{CV} \%$ & & 10.372 & 12.207 \\
Canario & CD - RDC & $2.37 \mathrm{a}^{\mathrm{z}}$ & $2.90 \mathrm{a}^{\mathrm{z}}$ \\
& CC - Control & $2.29 \mathrm{a}$ & $2.87 \mathrm{a}$ \\
$\mathrm{CV} \%$ & & 12.583 & 16.638 \\
\hline
\end{tabular}

${ }^{\mathrm{z}}$ Valores medios de tratamiento con la misma letra dentro de columnas son iguales de acuerdo con la prueba de Fisher a una $\mathrm{P} \leq 0.05$.

CV: Coeficiente de variación.

\section{Crecimiento de fruto}

El perfil de crecimiento presentó una curva con forma doble sigmoidal, con una fase I de crecimiento moderado hasta el 08 de noviembre, una fase II de crecimiento lento de catorce días, hasta el 22 de noviembre, y una fase III de crecimiento muy rápido hasta cosecha para la variedad Florida 39 y para la variedad Canario, el perfil de crecimiento presentó una curva con forma doble sigmoidal, con una fase I de crecimiento moderado hasta el 18 de octubre, una fase II de crecimiento lento de veintisiete días, hasta el 14 de noviembre, y una fase III de crecimiento muy rápido hasta cosecha (Figura 2).

El crecimiento de fruto durante las fases I y III de ambas variedades se caracterizó por un aumento rápido de volumen de las células del mesocarpio y, la disponibilidad de agua para las etapas antes mencionadas no afectó el tamaño final del fruto respecto al tratamiento control puesto que no hubo diferencias significativas. 

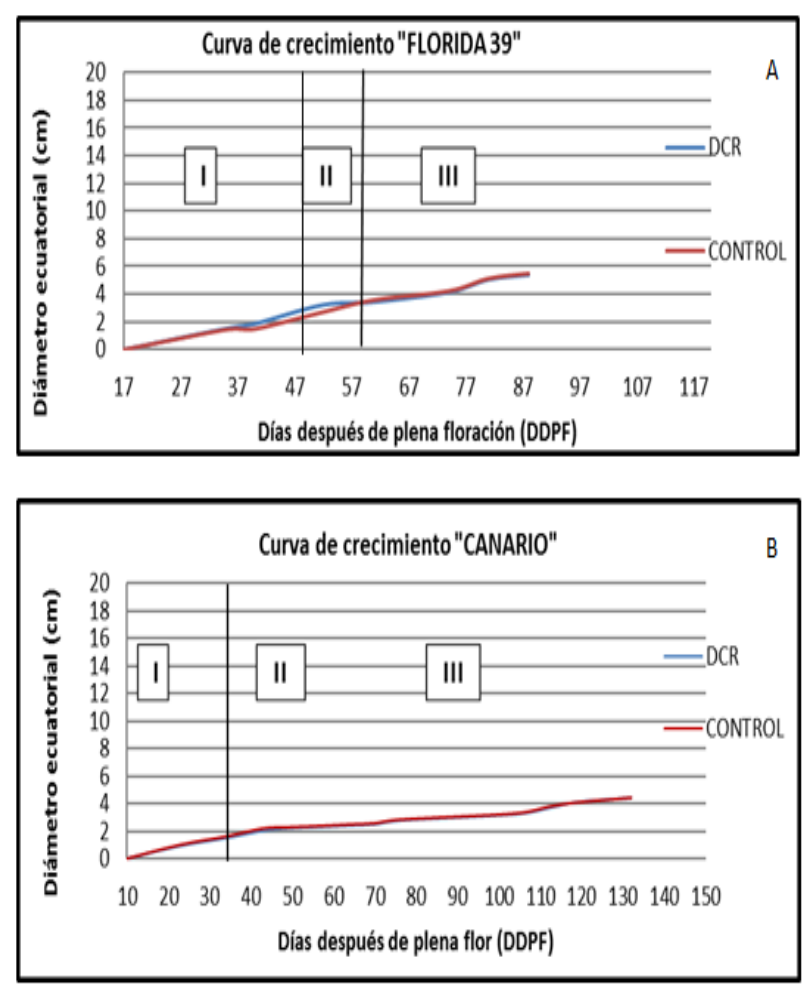

Figura 2. Perfil de crecimiento para la variedad Florida 39 (A) y perfil de crecimiento para la variedad Canario (B).

\section{Rendimiento y sus componentes}

Los tratamientos de riego deficitario no tuvieron repercusiones negativas sobre la producción total de fruta, no observándose diferencias significativas en ninguno de los dos tratamientos. Tampoco hubo diferencias significativas en número de frutos por árbol. La producción media aproximada para la variedad

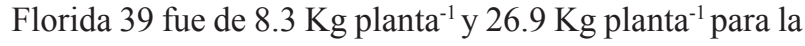
variedad Canario. Se detectaron diferencias numéricas en las producciones de ambas variedades, debido a tratarse de la primera cosecha en cultivares re injertados con menos de dos años, asimismo hubo problemas de falta de frío durante los meses de invierno, lo que provoco caída de yemas de flor, así como un bajo e irregular cuaje de frutos.

A pesar del aumento de la densidad de flores en el tratamiento RDC el rendimiento por planta no aumentó significativamente con respecto al control. Estos resultados son similares a los reportados por Li et al en 1989; Williamson y Coston en 1990; Boland en la bibliografía año1993 y Girona et al en 1993 los cuales concluyen que un déficit hídrico en la fase II de crecimiento del fruto del duraznero no afectan significativamente los rendimientos. Respecto al peso de fruto, Tabla 7, tampoco se observaron diferencias significativas entre los tratamientos de riego.

Las diferencias en volúmenes de agua de riego aplicados en la variedad Canario fueron notables, por lo que la eficiencia en el uso del agua, referida a kilogramos de fruta producida por $\mathrm{m}^{3}$ de agua fue significativa, desde $16.94 \mathrm{Kg} / \mathrm{m}^{3}$ de fruta en el menos regado a $13.55 \mathrm{Kg} / \mathrm{m}^{3}$ en el tratamiento control. Por otro lado, la variedad Florida 39 no presentó diferencia significativa en la eficiencia del uso del agua, obteniéndose $4.70 \mathrm{~kg} / \mathrm{m}^{3}$ de fruta en el tratamiento RDC y $4.44 \mathrm{Kg} / \mathrm{m}^{3}$ en el tratamiento Control.

Dicho resultado coincide con Mitchell y Chalmers (Citado por Podesta, 2007), puesto que encontraron que la EUA expresada como rendimiento por unidad de irrigación aumentó de 4.9 a $8.0 \mathrm{Kg} . \mathrm{m}^{-}{ }^{3}$ en durazno de industria con RDC con un rendimiento promedio de 48 ton.ha- ${ }^{-1}$.

Tabla 7. Rendimiento del durazno variedades Canario y Florida 39.

\begin{tabular}{llccc}
\hline & & Peso de fruto $(\mathrm{g})$ & Rendimiento por planta $(\mathrm{kg})$ & Eficiencia en el uso de agua $\left(\mathrm{Kg} / \mathrm{m}^{3}\right)$ \\
\hline Florida 39 & FD - RDC & $126.52 \mathrm{a}^{\mathrm{z}}$ & $7.899 \mathrm{a}^{\mathrm{z}}$ & $4.703 \mathrm{a}^{\mathrm{z}}$ \\
CV \% & FC - Control & $142.25 \mathrm{a}$ & $8.691 \mathrm{a}$ & $4.436 \mathrm{a}$ \\
Canario & 27.313 & 27.273 & 24.549 \\
CV \% & CD - RDC & $50.315 \mathrm{a}^{\mathrm{z}}$ & $27.240 \mathrm{a}^{\mathrm{z}}$ & $16.943 \mathrm{a}^{\mathrm{z}}$ \\
\hline
\end{tabular}

${ }^{\mathrm{z}}$ Valores medios de tratamiento con la misma letra dentro de columnas son iguales de acuerdo con la prueba de Fisher a una $\mathrm{P} \leq 0.05$.

$\mathrm{CV}$ : Coeficiente de variación.

Las evaluaciones de los frutos (altura (h) y diámetro ecuatorial $(\varphi \mathrm{E}))$ no tuvieron diferencias significativas entre los tratamientos de riego (Tabla 8).
Tabla 8. Evaluación de los frutos cosechados.

\begin{tabular}{llcc}
\hline & & $\varphi_{\mathrm{F}}(\mathrm{cm})$ & $\mathrm{h}(\mathrm{cm})$ \\
\hline Florida 39 & FD - RDC & $5.41 \mathrm{a}^{\mathrm{z}}$ & $5.94 \mathrm{a}^{\mathrm{z}}$ \\
& FC - Control & $5.50 \mathrm{a}$ & $6.32 \mathrm{a}$ \\
CV \% & & 3.928 & 11.084 \\
Canario & CD - RDC & $4.48 \mathrm{a}^{\mathrm{z}}$ & $4.66 \mathrm{a}^{\mathrm{z}}$ \\
& CC - Control & $4.43 \mathrm{a}$ & $4.69 \mathrm{a}$ \\
CV \% & \multicolumn{4}{c}{10.4} & 8.054 \\
\hline ZValores medios de tratamiento con la misma letra dentro de columnas \\
son iguales de acuerdo con la prueba de Fisher a una P $\leq 0.05$. \\
CV: Coeficiente de variación.
\end{tabular}


La cosecha de los frutos de la variedad "Canario" puso de manifiesto que la producción media en el tratamiento RDC no fue significativa en comparación con tratamiento Control. Numéricamente alcanzó una cifra mayor que en los árboles del tratamiento control (Tabla 7). Esta diferencia se debió a que los frutos de los árboles del tratamiento RDC contaban con un mayor peso medio entre grandes y medianos, asimismo, el número de frutos cosechados por árbol no varió en ambos tratamientos. Puede inferirse que con un déficit hídrico desde la floración hasta la fructificación, los frutos pueden alcanzar un tamaño final similar e incluso mayor en comparación con el tratamiento Control .Cabe mencionar que la distribución de frutos por tamaño se vio favorecida de tal forma que en el tratamiento RDC había un porcentaje mayor de frutos en los tamaños mayores de 3.8 a $4.3 \mathrm{~cm}$ (Figura3).

Para el caso de la variedad "Florida 39", la producción media en el tratamiento RDC no fue significativa en comparación con el tratamiento control. En este el fruto contaba con un menor peso medio en el tratamiento RDC y el número de frutos por árbol no varió entre los dos tratamientos (Tabla 7). La distribución de frutos por tamaños se vio favorecida de tal forma que en el tratamiento RDC había un porcentaje mayor de frutos en los tamaños mayores de 5,00 cm en comparación con los del tratamiento Control.

Un aspecto importante en el valor de la cosecha fue la distribución de los calibres. En variedades tempranas según Prieto et al., (2005) se admiten como comerciales calibres menores que en variedades de media estación o tardía. En la Figura se aprecia que no existió un efecto claro por tratamiento sobre la distribución de calibres y, por lo tanto, a priori no parece existir un cambio en el valor de la cosecha como consecuencia de la aplicación de tratamientos de riego deficitario.

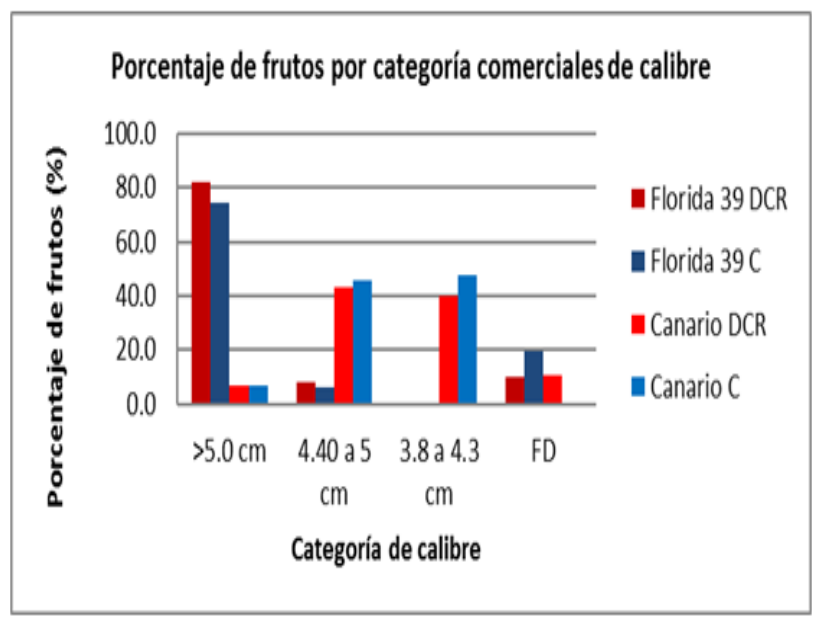

Figura 3. Porcentaje de frutos por categorías comerciales de calibre en cosecha de durazno 'Florida 39' y 'Canario' luego de los tratamientos de riego.
El tratamiento de restricción hídrica no afectó el calibre de los frutos de los tratamientos, con respecto a la variedad Florida 39 , más del $50 \%$ de los frutos presentaron calibre mayor a $5.0 \mathrm{~cm}$ y entre un 10 y $15 \%$ de frutos presentaron calibre 4,40 a $5 \mathrm{~cm}$. No obstante, para la variedad Canario, más del $40 \%$ de los frutos presentaron calibre entre 3,80 a $5 \mathrm{~cm}$ y 5 a $8 \%$ presentaron calibre mayor a $5 \mathrm{~cm}$.

Para la variedad Florida 39 resultó el tratamiento RDC, $0.881 \mathrm{~mm}$ de menor calibre de diámetro ecuatorial y 3,789 mm de menor calibre en la altura en comparación con el tratamiento C. Sin embargo para la variedad Canario el diámetro ecuatorial con el tratamiento RDC fue mayor en $0.452 \mathrm{~mm}$ con respecto al tratamiento $\mathrm{C}$, no obstante para la altura, el tratamiento $\mathrm{C}$ fue mayor en $0.271 \mathrm{~mm}$ respecto al tratamiento RDC (Figura 4).

Estos resultados concuerdan con Boland et al (1993) y Li et al (1989), los cuales indican, que niveles reducidos de agua en la fase II de desarrollo del fruto, no afecta el tamaño final del fruto, presentando estos una recuperación en su tasa de crecimiento una vez repuesto el régimen de riego normal $(100 \%$ Etc). El crecimiento del fruto se ve afectado significativamente cuando los árboles son sometidos a un menor suministro hídrico durante la tercera fase de crecimiento del fruto Li et al (1989).
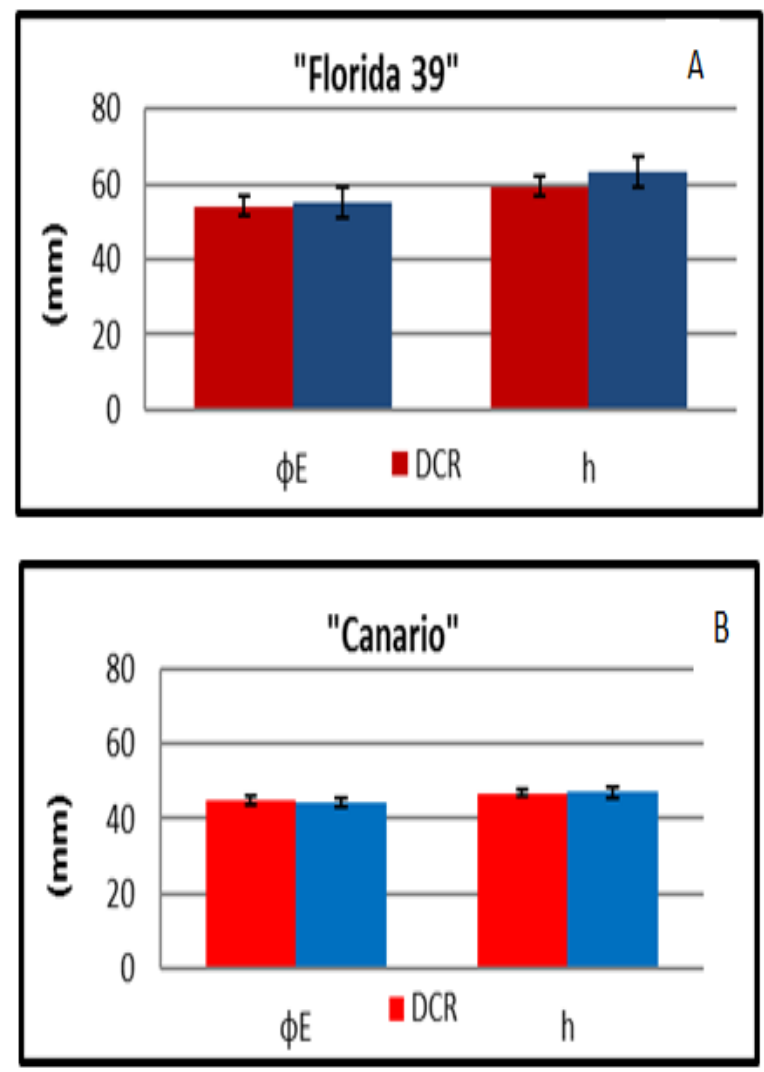

Figura 4. Evaluación de poscosecha (altura (h) y diámetro ecuatorial $\left(\varphi_{\mathrm{E}}\right)$ ) en los cultivares Florida 39 (A) y Canario (B). 
Los datos de clasificación por calibre de los frutos están reflejados en el Tabla 9. El porcentaje de fruta de tamaño extra ( $>5.0 \mathrm{~cm}$ de diámetro ecuatorial) para la variedad Florida 39, se incrementó en casi 1.1 veces al aplicar el $\mathrm{RDC}$ en relación a los árboles testigos $(\mathrm{C})$ aun cuando no fue significativo. Sin embargo, para la variedad Canario, el porcentaje de fruta de tamaño extra $(>5.0 \mathrm{~cm}$ de diámetro ecuatorial) se redujo en 1.03 veces al aplicar el $\mathrm{RDC}$ en comparación con los árboles testigo.

Tabla 9. Clasificación por calibre de los frutos.

\begin{tabular}{|c|c|c|c|c|}
\hline \multirow{4}{*}{ Fuentes de variación } & \multicolumn{4}{|c|}{ Distribución de la fruta \% } \\
\hline & & 1 & 2 & \multirow{3}{*}{$\mathrm{FD}^{*}$} \\
\hline & \multicolumn{3}{|c|}{$>5.0 \mathrm{~cm}(4.4-5.0)(3.8-4.3)$} & \\
\hline & & $\mathrm{cm}$ & $\mathrm{cm}$ & \\
\hline Florida FD - RDC & 81.915 & 8.2979 & --- & 9.787 \\
\hline $39 \quad \mathrm{FC}-$ Control & 174.584 & 5.9382 & --- & 19.48 \\
\hline \multirow{2}{*}{ Canario $\begin{array}{l}\text { CD - RDC } \\
\text { CC - Control }\end{array}$} & 6.51 & 43.021 & 39.648 & 10.821 \\
\hline & 6.712 & 45.75 & 47.509 & 0.029 \\
\hline
\end{tabular}

\section{Conclusiones}

De los resultados obtenidos se puede concluir que para la variedad Florida 39, la cantidad de agua total recibida fue $369.111 \mathrm{~mm}$ en el tratamiento Control y $316.667 \mathrm{~mm}$ en el tratamiento RDC, y para la variedad Canario, la cantidad total recibida fue $369.111 \mathrm{~mm}$ en el tratamiento Control y $303 \mathrm{~mm}$ en el tratamiento RDC, lo que significa un ahorro de $17.91 \%$ (RDC) y $14.21 \%$ (RDC) de agua durante todo el ciclo de cultivo respecto del tratamiento control (C) regado con un $100 \%$ de la ETc para las variedades Florida 39 y Canario respectivamente.

Para las variedades Florida 39 y Canario, la eficiencia de uso del agua a nivel de planta, fue mayor sólo para el tratamiento más restrictivo $\mathrm{RDC}\left(4.70 \mathrm{Kg} / \mathrm{m}^{3}\right.$ y $16.94 \mathrm{Kg} /$ $\mathrm{m}^{3}$, respectivamente) respecto al tratamiento $\mathrm{C}(4.44 \mathrm{Kg} /$ $\mathrm{m}^{3}$ y $13.55 \mathrm{Kg} / \mathrm{m}^{3}$, respectivamente), siendo el efecto de riego no significativo para esta variable en la variedad Florida 39 y significativo en la variedad Canario.

El tratamiento RDC, no afectó significativamente el crecimiento de los árboles y la sección transversal de las ramas, asimismo el tratamiento RDC no afecto el calibre y peso medio de los frutos, presentando la variedad Florida 39, frutos de aproximadamente 142.25 gramos para el tratamiento control y frutos de 126.52 gramos para el RDC. En el caso de la variedad Canario éste presentó frutos y de aproximadamente 49.430 gramos para el tratamiento Control y 50.315 gramos para el RDC.

La mayor respuesta productiva del melocotón para la variedad Florida 39, en cuanto al rendimiento total $(8.691 \mathrm{Kg} /$ planta) se obtiene cuando es regado sin déficit hídrico durante todo su ciclo vegetativo, la aplicación del tratamiento RDC no condiciona sensiblemente la producción (7.899 Kg/planta). Caso contrario ocurre con la variedad Canario, donde los resultados demuestran que la mayor respuesta productiva de melocotón (27.240 Kg/ planta), se obtiene cuando es regado con el tratamiento RDC. Aplicando un riego con tratamiento Control, la producción fue de $(26.537 \mathrm{Kg} /$ planta $)$.

\section{Literatura citada}

Alarcón, J.J., Torrecillas, A., Sánchez blanco, M.J., Abrisqueta, J.M., Vera, J., Pedrero, F., Magaña, I., García-Orellana, Y., Ortuño, M.F., Nicolás, E., Conejero, W., Mounzer, O., Ruíz, M.C. 2006. Estrategias de riego deficitario en melocotonero temprano. Vida Rural, No 225. Págs.28-32.

Blanco, O., González, J.M., Espada, J.L. 2010. Respuesta del melocotonero de Calanda al riego deficitario. Comunicación A-08. En: El regadío: una apuesta por el desarrollo del medio rural, XXVIII Congreso Nacional de Riegos, León. 8pp.

Boland, A., Mitchell, P., Goodwin, I. 1993. The effect of regulated deficit irrigation on tree water use and growth of peach. J. of Hortic. Sci. 68:261-274.

Ferreyra, R., Selles, G., Lemus, G. 2002. Efecto del estrés hídrico durante la fase II de crecimiento del fruto del duraznero cv. Kakamas en el rendimiento y estado hídrico de las plantas. Agricultura Técnica (Chile) 62 . Págs.565-573.

Girona, J., M. Mata, D. Goldhamer, R. Johnson, and T. De Jong. 1993. Patterns of soil and tree water status and leaf functioning during regulated deficit irrigation scheduling in peach. J. Am. Soc. Hortic. Sci. 118:580586.

Girona, J. 1994. Respuesta fisiológica y productiva del estrés hídrico en diferentes especies frutales: Melocotonero (Prunus pérsica L Batsch), Avellano (Corylus avellana L) y nogal (Juglans regia). Tesis para optar el grado de Doctor Ingeniero Agrónomo por la Universidad de Lleida. Escuela técnica superior de Ingeniería Agraria. 151pp.

Larson, K.D.; T.M. Dejong Y R.S. Johnson. 1988. Physiological and growth responses of mature peach trees to postharvest water stress. J. Amer. Soc. Hort. Sci. 113(3):296-300.

Li, S., J. Huguet, P. Schoch, and P. Orlando. 1989. Response of peach tree growth and cropping to soil water deficit at varius phenological stages of fruit development. J. Hortic. Sci. 64:541-552.

Martinez, A., Romero, P., Fernández, J. 2007. Técnicas de riego deficitario en el cultivo de la vid. Vida Rural (244). Págs. 58-64

Merriam, J.L; Keller, J. 1978. Farm irrigation systems evaluation. A guide for management, 3er edition. Agricultural and Irrigation Engineering Department, Utah State University. Logan, USA. 235p. 
Podestá, L. 2007. Influencia de restricciones hídricas poscosecha en el crecimiento vegetativo y reproductivo en plantaciones jóvenes de cerezo (Prunus avium L.). Tesis para optar el grado de Magister Scientiae en Riego y Drenaje por la Universidad Nacional de Cuyo. Facultad de Ciencias agrarias. 99pp.

Prieto, M.H., Moñino, M.J., Vivas, A. 2005. Estrategias de riego deficitario en melocotón temprano. Vida Rural, No 206. Págs.38-42.

Ruiz Sánchez, M.C., Girona, J. 1995. Investigaciones sobre riego deficitario controlado en melocotonero. En: Riego Deficitario Controlado. Cuadernos VALUE, Mundi-Prensa S.A., Madrid. Págs.69-95.

Sánchez Blanco, M.J., Torrecillas, A. 1995. Aspectos relacionados con la utilización de estrategias de riego deficitario controlado en cultivos leñosos. En: Riego Deficitario Controlado. Cuadernos VALUE, MundiPrensa S.A., Madrid. Págs.45-65.

Williamson, J.G., and D.C. Coston. 1990. Planting method and irrigation rate influence vegetative and reproductive growth of peach planted at high density. J Am. Soc Hortic. Sci. 115:207-212. 